\title{
Compact Photonic Transmitter Based on Annular Ring Antenna for THz Applications
}

\author{
Ibtissame Moumane*1, Jamal Zbitou ${ }^{2}$, Ahmed Errkik ${ }^{3}$, Larbi El Abdellaoui ${ }^{4}$, \\ Abdelali Tajmouati ${ }^{5}$, Mohamed Latrach ${ }^{6}$ \\ 1,2,3,4,5 LMEET, University of Sciences Hassan 1St University of Settat Morocco, Morocco \\ ${ }^{6}$ Microwave groupe ESEO, Angers, France \\ *Corresponding author, e-mail: moumaneibti@gmail.com¹, zbitou3676@gmail.com²
}

\begin{abstract}
This paper presents the design of Continuous Wave Terahetz photonic transmitters based on photodector which convert the light signal to electrical signal, THz antenna, low-pass filter (LPF) and DC Probe. In the design of the $\mathrm{CW} \mathrm{THz} \mathrm{photonic} \mathrm{transmitter} \mathrm{System,} \mathrm{we} \mathrm{begin} \mathrm{with} \mathrm{the} \mathrm{matching} \mathrm{input}$ impedance and validation of $\mathrm{THz}$ antenna using an EM solver Momentum integrated in ADS "Advanced Design System". Then we pass to the optimization of a low-pass filter which had the role of inductance, blocking the RF signal providing from the antenna to reach the DC probe. Finally, we associate the previous structures with a DC probe and simulate the whole circuit until validating the CW THz photonic transmitter circuit. The three structures are based on multi-layers GaAs substrate, which is the most widely used for $\mathrm{THz}$ circuit design. The dimensions of the whole circuit are $819.071 \times 164.10 \mathrm{\mu m}^{2}$.
\end{abstract}

Keywords: GaAssubstarte, CW THz photonic transmitter, photodetector, antenna THz, low-pass filter

Copyright $\odot 2018$ Universitas Ahmad Dahlan. All rights reserved.

\section{Introduction}

Recently, weremark the fastgrowinginterestgiving to the THzdomain. A diversity of domainssuch as biomedicalimaging, spectroscopy, Security and telecommunications are focusingnowtheirs applications on THzwaveswhichpresentseveraladvantagesbased on interactivitywith the materialwhereitspreads and fast absorption by the atmosphere [1]. Also the apparition of the modern Femtosecond Lasers and High-Speed Photodetectorsgive the opportunity to develop more research in the generation, detection and guiding of THzwaves in the aim to obtain a compact high-power and high-efficiencyTHztransmitter.To generate the THzwavesmanymethods are proposed but the mostusedis the one thatrelays on the coplanarwaveguide (CPW) photonictransmitters [2].

The CPW technologyoffers in factseveraladvantages due to itsfeatures, likelow radiation, low dispersion, easy of shunts and series connections [3].This paperpresents a new design of a $\mathrm{CW} \mathrm{THz}$ photonic transmitter composed from a photo detector associated to $\mathrm{THz}$ antenna inserts in serieswith a lowpassfilter and a DC Probe. Weevoke the theory of different components of the system, then the design and validation of the proposed $\mathrm{THz}$ transmitter by using Momentum electromagnetic solver.

\section{Photonic Transmitter System}

\subsection{THz Technology}

As mentioned before $\mathrm{THz}$ technology attracts more and more searchers. As result it's widen their areas of application and give birth to others. The THz frequency is between microwave and visible waves as illustrated in the Figure 1 and occupies the $100 \mathrm{GHz}-10 \mathrm{THz}$ spectrum. The $\mathrm{THz}$ band has various advantages of the application summarized as follows [4]:

a. Microwave band is almost all preoccupied by different services, and its bandwidth is limited. In place of this, the terahertz can offer a wider bandwidth.

b. The diffraction of the $\mathrm{THz}$ wave is low in comparison with that of the microwave and millimeter wave, which is the advantageous in the line-of-sight (LOS) and point-to-point link.

c. This band offers high degree of information security, especially in the spread spectrum technology. 
d. In comparison with infrared, $\mathrm{THz}$ has low attenuation of the signal in certain atmospheric conditions like fog.

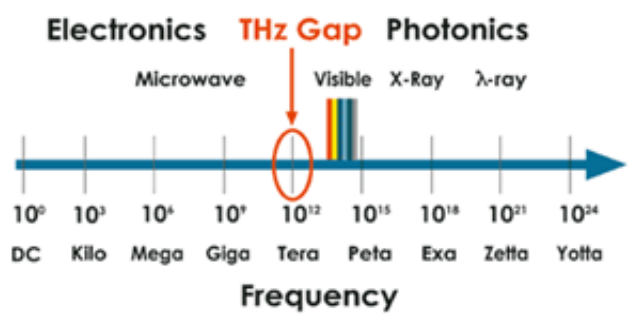

Figure 1. Position of $\mathrm{THz}$ band between the microwave and infrared regime of electromagnetic spectrum

\subsection{Photodetector "PD"}

A PD is a sensor its role is to convert an optical power into an electrical current. To generate electron-hole-pairs, the photon energy provides from the light absorbed in a PD must be at last equal to the bandgap energy $\mathrm{Eg}$ of the absorber material [5].This available energy of one photon is sufficient to excite an electron from the valence band (v.b.) to the conduction band (c.b.). For this band-to-band transition, the upper wavelength limit for photon absorption is given by [5]:

$$
\lambda_{\mathrm{g}}[\mu \mathrm{m}]=\frac{1.24}{\mathrm{Eg}_{\mathrm{g}}[\mathrm{eV}]}
$$

A PD has different proprieties such as:

Sensitivity: The ability of the photodiode to transform light absorbed into an electrical current in other term the number of charge carrier pairs generated per incident photon [5].

$$
\eta_{\text {ext }}=\frac{I_{P d}}{q} \cdot \frac{h v}{P_{o p t}}
$$

Responsivity: where Ipd is the photogenerated current by the absorption of the optical input power Popt at a frequency $\vee$ mentied in equation (2). A common figure of merit is the external responsivity $R$, defined as the ratio of photocurrent to the input optical power [5]:

$$
\mathrm{R}=\frac{\mathrm{I}_{\mathrm{Pd}}}{\mathrm{P}_{\mathrm{opt}}}=\frac{\eta_{\text {ext }} \lambda[\mu \mathrm{m}]}{1.24} \mathrm{~A} / \mathrm{W}
$$

In this study we choose the Metal Semiconductor-Metal Traveling wave Photodetector (MSM-TPD) due to its high power-bandwidth and coplanar-waveguide fed slot owing to its easy connection with planar devices [6]. The PD based on GaAs substrate which characterized by a succession of layers as mentioned in the Figure 2 [7]:

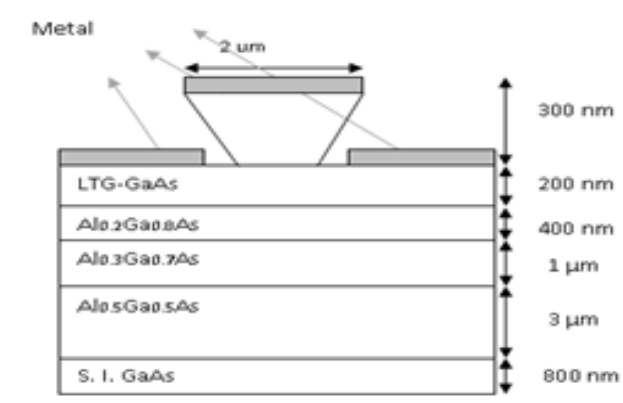

Figure 2. Structure of the photodetector based on GaAs substrate 


\subsection{THz Antenna}

The role of the antenna [8-10] is to transmit the RF signal providing from PD. It presents one of the most important element in the design of CPW THz photonic transmitter. The proposed antenna illustrated in Figure 3 presents an annular ring shape [11-13]. Table 1 shows values of design parameters (Unit in $\mu \mathrm{m}$ ).

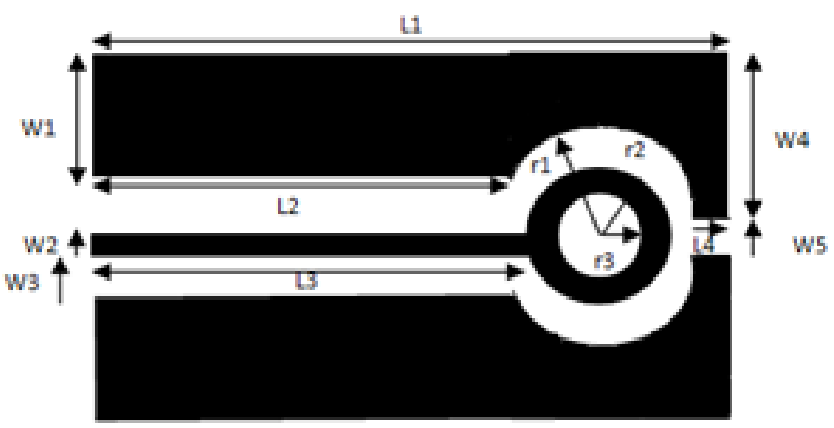

Figure 3. The THz annular ring proposed antenna

Table 1. Values of Design Parameters (Unit in $\mu \mathrm{m}$ )

\begin{tabular}{cc}
\hline Dimensions $(\mu \mathrm{m})$ & Values \\
\hline L1 & 176.60 \\
L2 & 121.42 \\
L3 & 126.61 \\
L4 & 5 \\
r1 & 25.97 \\
r2 & 21.64 \\
r3 & 36.34 \\
W1 & 19.39 \\
W2 & 5.6 \\
W3 & 11.06 \\
W4 & 28.41 \\
W5 & 6.01 \\
\hline
\end{tabular}

The result of S11 presenting in Figure 4 makes the proposed antenna suitable for $\mathrm{THz}$ $\mathrm{CW}$ photonic transmitters. The reflection coefficient is below $-10 \mathrm{~dB}$ between the frequency 1.98 $\mathrm{THz}$ and $2.02 \mathrm{THz}$. To obtain the radiation diagram which describes the behavior of the antenna we have launched a 3D simulation at $2 \mathrm{THz}$ in $\mathrm{ADS}$ as shown in Figure 5. As presented the radiation patter is multidirectional with stable radiation.

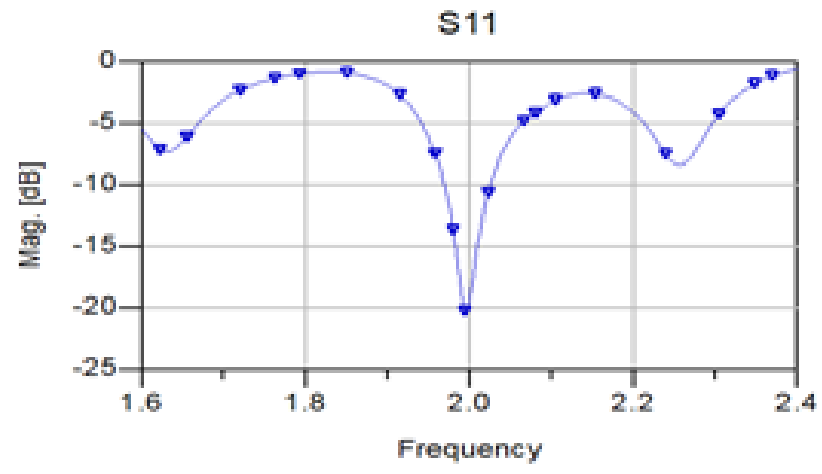

Figure 4. Reflection coefficient versus frequency

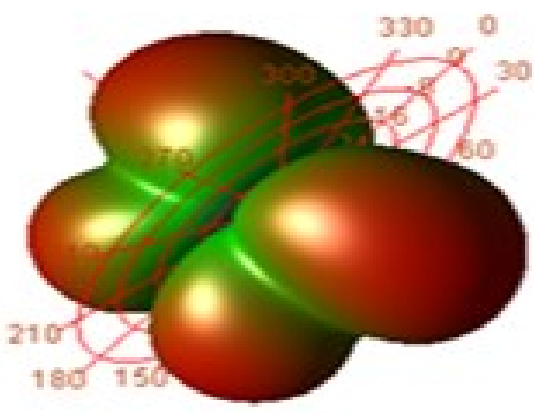

Figure 5. The radiation pattern at $2 \mathrm{THz}$ 


\subsection{Low-Pass Filter "LPF"}

To block the RF signal providing from PD and transmitting via antenna, to reach the DC probe the integration of Low Pass Filter into the CW photonic transmitter system permits to block the RF signal from reaching the DC probe. Figure 6 presents a several periodic structure composed from three units inspired from the study [14-15]. The Table2 resumes the different dimensions of the proposed LPF:

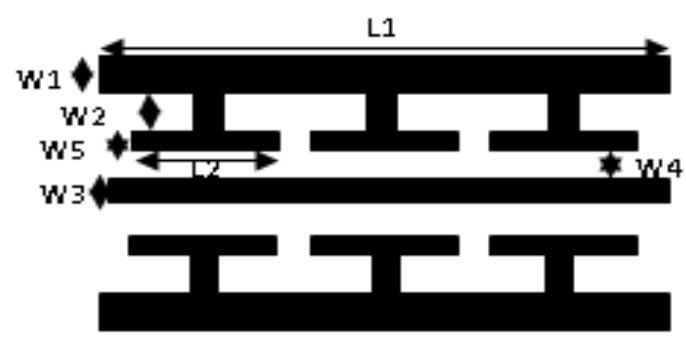

Figure 6. The layout of the periodic LPF THz structure

Table 2. Filter Dimensions (Unit in $\mu \mathrm{m}$ )

\begin{tabular}{cc}
\hline Dimensions $(\mu \mathrm{m})$ & Values \\
\hline L1 & 300 \\
L2 & 60 \\
W1 & 20 \\
W2 & 10 \\
W3 & 17 \\
W4=Gap & 5 \\
W5 & 5 \\
\hline
\end{tabular}

As presented in Figure 7 the LPF presents a low insertion loss, with a cutoff frequency of $0.45 \mathrm{THz}$ and a wide rejection band until $1.2 \mathrm{THz}$, The phase of $\mathrm{S} 21$ coefficient is presented in Figure 8.To study the behavior of the LPF structure, we have launched a simulation at $0.3 \mathrm{THz}$ in the frequency passband. In addition, at $1 \mathrm{THz}$ in the rejection band. As shown in Figure 10 the conclusion is the filter "LPF » playsitsrole, at0.3 THzunder the cutofffrequency the RF energypassfrom port 1 to port 2 and at $1.15 \mathrm{THz}$ the energyhadstoppedwhichimprovesthat the proposed LPF issuitable for THz system.Figure9 shows the current density @ (a) $0.3 \mathrm{THz}$ and $1 \mathrm{THz}$.

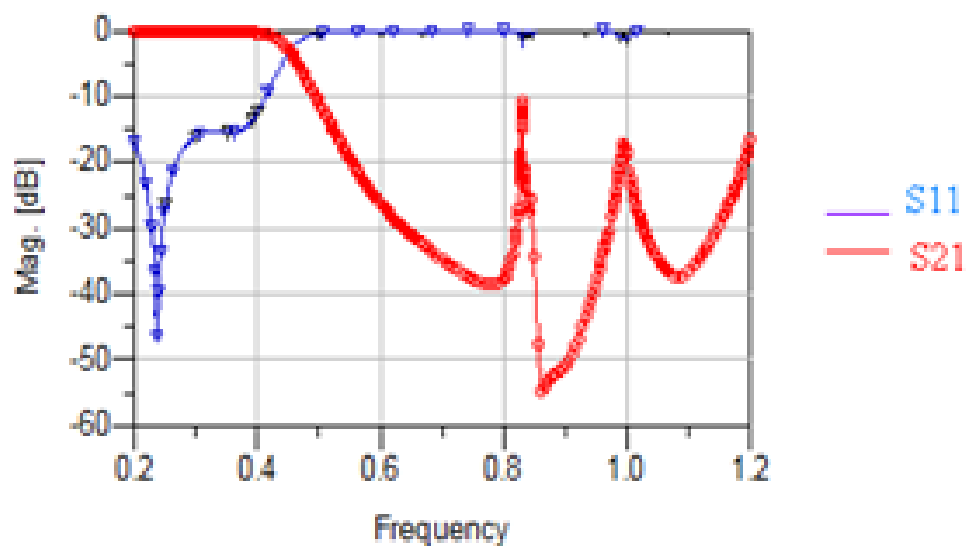

Figure 7. Simulation S-Parameters results versus frequency 


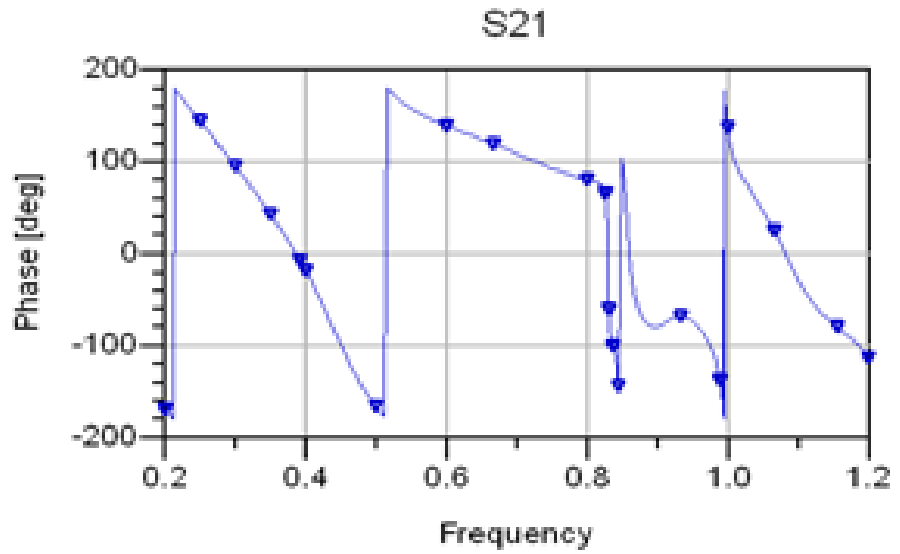

Figure 8. Phase of S21 versus frequency

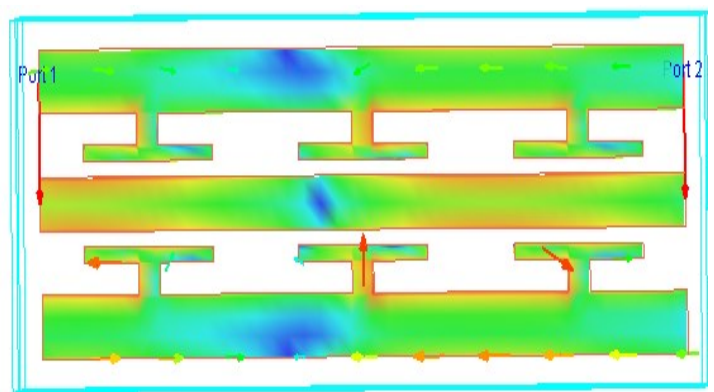

(a)

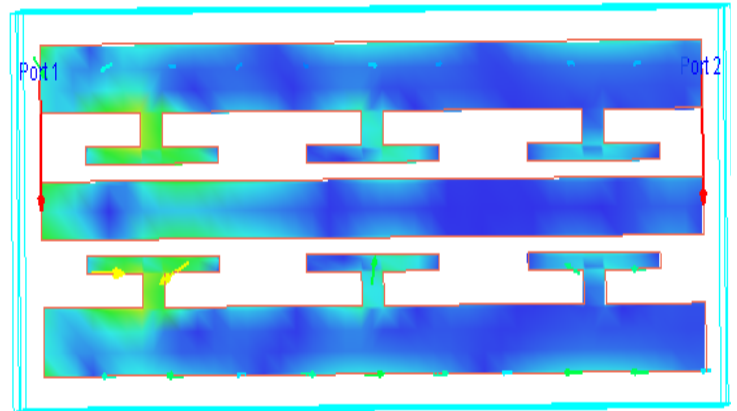

(b)

Figure 9.The current density @ (a) $0.3 \mathrm{THz}$ and $1 \mathrm{THz}$

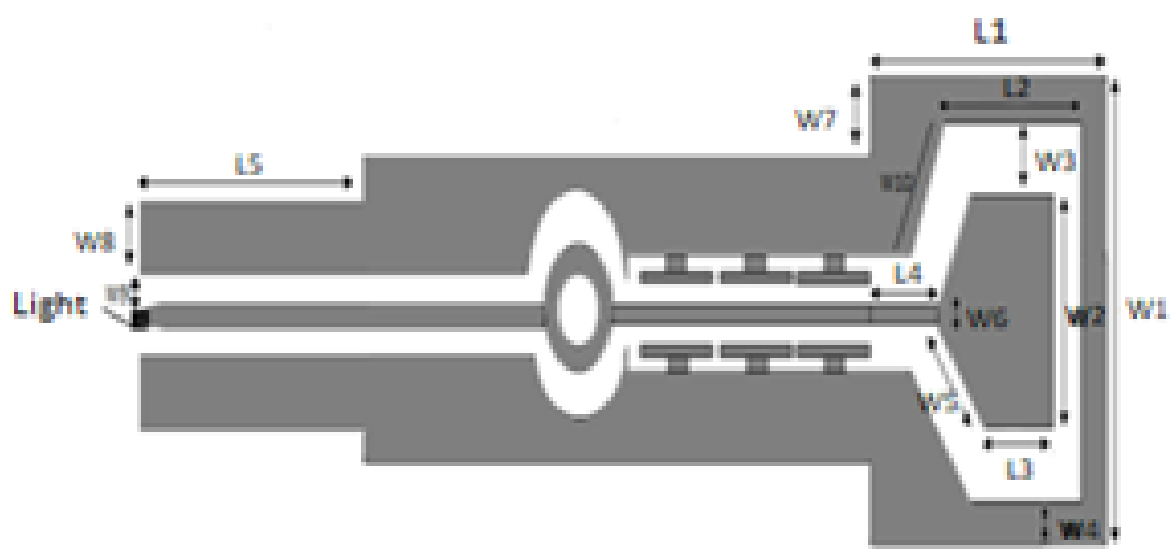

Figure 10. The proposed CW photonic transmitter

\subsection{CW Photonic Transmitter Simulation}

After presenting the annular ring THz antenna and the "LPF", we have associated the photodetector to the system composed from the antenna, the filter and DC probe responsible for the DC bias to obtain the CW photonic transmitter system. Table 3 showsvalues of DC probe parameters. 
The proposed photonic transmitter was firstly optimized for an input impedance equal to $50 \mathrm{ohm}$, the circuit presents a good matching input impedance as shown in Figure 11(a) with a narrow bandwidth. To take into account the input impedance of the photodetector around $30 \mathrm{Ohm}$ we have simulated the $\mathrm{THz}$ transmitter which permits to obtain a large matching input impedance between 1.51THz and 1.59THz.

Table 3. Values of DC Probe Parameters

\begin{tabular}{cc}
\hline Dimensions $(\mu \mathrm{m})$ & Values \\
\hline L1 & 238.11 \\
L2 & 114.95 \\
L3 & 98.52 \\
L4 & 83.13 \\
L5 & 107.76 \\
W1 & 163.18 \\
W2 & 104.68 \\
W3 & 17 \\
W4 & 10.26 \\
W5 & 71.88 \\
W6 & 12.31 \\
W7 & 33 \\
W8 & 15.42 \\
W9 & 6.67 \\
W10 & 61.58 \\
\hline
\end{tabular}

After the validation of this $\mathrm{THz}$ photonic transmitter into simulation we have done a comparison between the proposed $\mathrm{THz}$ antenna which is the key of the $\mathrm{THz}$ source and another $\mathrm{THz}$ antenna validated in literature. The Table 4 presents the comparison of the performances (dimensions, frequency bandwidth) between the proposed antenna and two others structures: As shown in this table, the proposed antenna presents good performances in term of bandwidth and length.

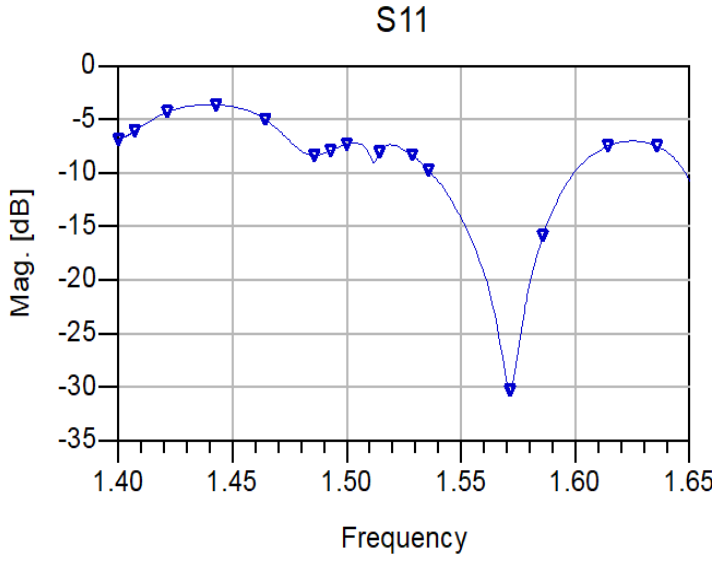

(a)

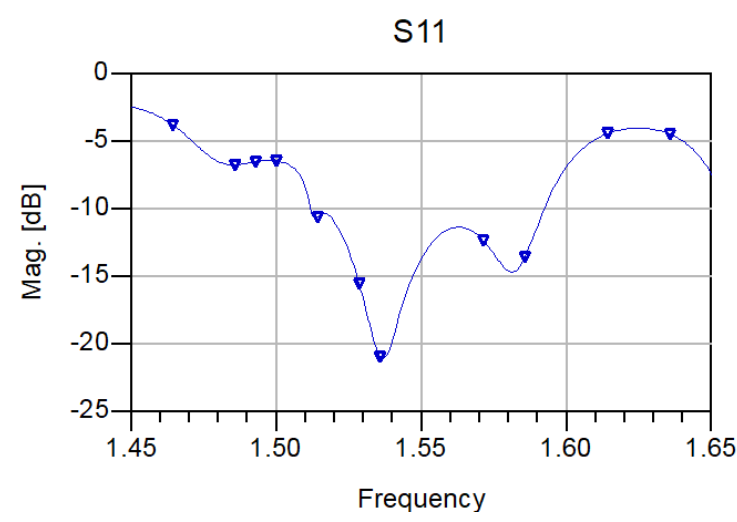

(b)

Figure11. Simulation S-Parameters results versus frequency (a) input impedance $50 \Omega$ (b) For $30 \Omega$ input impedance

Table 4. Comparison of the Antenna Structures

\begin{tabular}{ccc}
\hline $\begin{array}{c}\text { Antenna } \\
\text { Structure }\end{array}$ & Length & Frequency Bandwidth \\
\hline Proposed & $172.24 \mu \mathrm{m}$ & {$[1.98 \mathrm{Thz}, 2.02 \mathrm{THz}]$} \\
Antenna & $330 \mu \mathrm{m}$ & {$[1 \mathrm{THz}, 1.25 \mathrm{THz}]$} \\
Antenna[17] & $200 \mu \mathrm{m}$ & Narrow band at645 Ghz \\
Antenna [18] & $1040 \mu \mathrm{m}$ & Narrow bandat $850 \mathrm{Ghz}$ \\
Antenna [19] &
\end{tabular}




\section{Conclusion}

The aim of this paper is the validation of $\mathrm{CW}$ photonic transmitter using for generation of $\mathrm{THz}$ waves. As mentioned the $\mathrm{CW}$ photonic transmitter composed from Photodetector, Antenna, LPF and DC probe. Thus our study begin with the choice of Photodetector MSM-TPD which converts the light signal to electrical one, the optimization of the $\mathrm{THz}$ Antenna then the validation of low pass filter and finally we have associated all structures in series, the whole circuit was optimized and simulated using an electromagnetic solver Momentum integrated in ADS until reaching the desired results. The final circuit is mounted on a multilayer GaAs substrate and having an area around $819.071 \times 164.10 \mu \mathrm{m}^{2}$.

\section{References}

[1] Bertrand Gauvreau. Ondes terahertz.Ecole Polytechnique de Montréal, 2500 chemin de Polytechnique, Montréal, QC H3T 1J4, Canada.

[2] J Zbitou, C Minot, X Begaud, B Huyart.Bow-tie Wideband Antenna Design for CW THz Photonic Transmitters.Progress In Electromagnetics Research Symposium, Cambridge, USA, 2-6July 2008.

[3] S Khireddine, M Drissi, R Soares. Flat Group Delay Low Pass Filters Using Two CPW Topologies. IETR, CNRS UMR 6164, INSA Rennes, France, DAlightCom, Lannion, France.

[4] Kumud Ranjan Jha Ghanshyam Singh. Terahertz Planar Antennas for Next Generation Communication. Springer. January 2014.

[5] Vorgelegt von Diplom-Physiker Andreas Beling aus Bonn. Periodic Travelling Wave Photodetectors with Serial and Parallel Optical Feed Based on InP.

[6] ShiJW, KG Gan, YJ Chiu, YH Chen, CK Sun, YJ Yang, JE Bowers. Metal-semiconductor-metal traveling-wave photodetectors. IEEE Photonics Technology Let-ters, Jun 2001; 13(6): 623-625.

[7] Chi KuangSun. THz Optoelectronics. UltraFastOptics Laboratory (UFO) Graduate Institute of ElectroOptical Engineering and Department of Electrical Engineering National Taiwan University Taipei, Taiwan.

[8] KL Wong, CL Tang, HT Chen, A compact meandered circular microstrip antenna with a shorting pin. Microwave Opt. Technol. Lett. 20 June 1997; 15(3): 147-149,

[9] Ahmed El Hamraoui, El Hassane Abdelmounim, Jamal Zbitou, Laarbi Elabdellaoui, Hamid Bennis, Mohamed Latrach. A Dual-band Microstrip Slotted Antenna for UHF and Microwave RFID Readers TELKOMNIKA (Telecommunication, Computing, Electronics and Control). February 2018; 16(1): 94101.

[10] S El Kilani, L El Abdellaouib, J Zbitou, J Terhzaz, A Errkik, M Latrach. A Multiband Printed Antenna Suitable for Wireless Applications.TELKOMNIKA (Telecommunication, Computing, Electronics and Control).February 2018; 16(1): 159-165.

[11] KB Hsieh, MH Chen, KL Wong, Single-feed dual-band circularly polarized microstrip antenna, Electronics Letters. 11 June 1998; 34(12): 1170-1171.

[12] ZN Chen, MJ Ammann, MYW Chia,TSP.See Circular annular planar monopoles with EM coupling. IEE Proceedings-Microwaves, Antennas and Propagation. 8 Aug. 2003; 150(4): 269 - 273.

[13] Jean Yves Dauvignac,Nicolas Fortino,Stéphane Tourette,Georges Kossiavas,Pascal Ciais Miniaturisation des antennes UWB planaires.University Nice 25 October 2006.

[14] I Moumane, J Zbitou, J Terhzaz. AErkik, MLatrach. A Low-Pass Periodic Filter for CW THz Photonic Transmitters. WITS-ENSA of Kenitra 21-22 April 2016.

[15] James Sor, YongxiQian, Tatsuo Itoh. Miniature Low-Loss CPW Periodic Structures for Filter Applications.IEEE Transactions on Microwave Theory and Techniques. December 2001; 49(12): 23362341.

[16] Advanced Design System : https://www.keysight.com/

[17] I Moumane, J Zbitou, A Erkik. M Latrach, O Chakkor, A Fouad. Design of CW THz Photonic transmitter based on Low Pass-Filter and Bow-tie Wideband Antenna.International Journal of Electrical and Computer Engineering (IJECE). October 2018; 8(5): 3801-3808.

[18] Ming Chun Tien, Hsu Hao Chang, Ja Yu Lu, Li Jin Chen, Shih Yuan Chen, Ruey Beei Wu, Wei Sheng Liu, Jen Inn Chyi, Chi Kuang Sun. Device Saturation Behavior of Submillimeter-Wave Membrane Photonic Transmitters. IEEE Photonics Technology Letters. March 2004; 16(3): 873-875.

[19] Yi Chun Chen, An Shyi Liu, Shih Yuan Chen, Ruey Beei Wu, Chi Kuang Sun. Design of Rampart Slot Array Antenna in Integrated $850 \mathrm{GHz}$ Photonic Transmitter. 2005 Asia-Pacific Microwave Conference Proceedings.4-7 Dec 2005. 